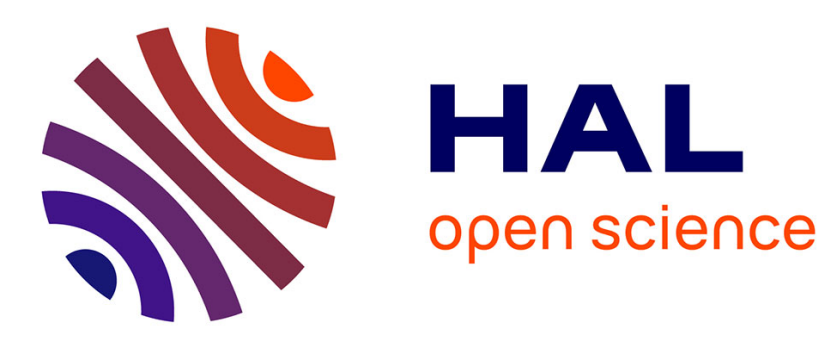

\title{
Inter-Landau level scattering and LO-phonon emission in Terahertz quantum cascade laser
}

\author{
Nicolas Péré-Laperne, Louis-Anne de Vaulchier, Yves Guldner, Gérald \\ Bastard, Giacomo Scalari, Marcella Giovannini, Jérôme Faist, Angela \\ Vasanelli, Sukhdeep Dhillon, Carlo Sirtori
}

\section{To cite this version:}

Nicolas Péré-Laperne, Louis-Anne de Vaulchier, Yves Guldner, Gérald Bastard, Giacomo Scalari, et al.. Inter-Landau level scattering and LO-phonon emission in Terahertz quantum cascade laser. Applied Physics Letters, 2007, 91, pp.062102. hal-00156810

\section{HAL Id: hal-00156810 https://hal.science/hal-00156810}

Submitted on 22 Jun 2007

HAL is a multi-disciplinary open access archive for the deposit and dissemination of scientific research documents, whether they are published or not. The documents may come from teaching and research institutions in France or abroad, or from public or private research centers.
L'archive ouverte pluridisciplinaire HAL, est destinée au dépôt et à la diffusion de documents scientifiques de niveau recherche, publiés ou non, émanant des établissements d'enseignement et de recherche français ou étrangers, des laboratoires publics ou privés. 


\title{
Inter-Landau level scattering and LO-phonon emission in Terahertz quantum cascade laser
}

\author{
N. Péré-Laperne, L.A. de Vaulchier, ${ }^{*}$ Y. Guldner, and G. Bastard \\ Laboratoire Pierre Aigrain, Ecole Normale Supérieure, 24 rue Lhomond, 75231 Paris Cedex 05, France \\ G. Scalari, M. Giovannini, and J. Faist \\ Institute of Physics, University of Neuchatel, CH-2000 Neuchatel, Switzerland
}

A. Vasanelli, S. Dhillon, and C. Sirtori

Laboratoire Matériaux et Phénomènes Quantiques,

Université Denis Diderot Paris VII, F-75005 Paris, France

(Dated: June 22, 2007)

\begin{abstract}
A Terahertz Quantum Cascade Laser (THz QCL) structure based on a bound to continuum and LO-phonon extraction stage is studied under a strong magnetic field. Two series of power oscillations as a function of magnetic field are observed. Comprehensive simulations of the lifetimes allow the first series to be assigned to interface roughness (elastic) and the second to LO phonon scattering (inelastic) of hot carriers in an excited Landau level, previously unobserved in terahertz QCL. We demonstrate that for the latter, the thermal electron distribution in the upper laser state leads to a breaking of the population inversion and hence laser action.
\end{abstract}

Quantum cascade lasers operating in the terahertz domain have recently attracted considerable attention, owing to the fact these unipolar semiconductor lasers are the only compact and coherent solid-state sources available in this frequency range. The observation of laser action below the reststrahlen band of GaAs ( $\hbar \omega_{L O} \sim 36 \mathrm{meV}$ ) was made possible by various designs of the active region in order to obtain population inversion between two levels closely spaced in energy. The combination of a long lifetime and good injection efficiency of the upper state with an efficient extraction of the lower state is the major challenge in order to obtain reasonable output power and high operating temperature. The first $\mathrm{THz}$ QCLs were based on chirped superlattice structures in which the laser transition occurs between the states of a superlattice minigap ${ }^{1,2}$ while, more recently, 'bound-to-continuum' designs have been studied ${ }^{3,4}$. In this latter design, electrons are injected into an isolated state and a miniband is employed as extraction mechanism to reduce the lower state lifetime. Designs using LO-phonon scattering for electron depopulation of the lower state have also been realized ${ }^{5-7}$. Such a depopulation mechanism offers the advantage to be extremely fast and has allowed the highest working temperatures. Recently, G. Scalari and co-authors ${ }^{8}$ significantly improved the performances of a bound to continuum $\mathrm{THz}$ laser by realizing a structure in which the extraction miniband is coupled to a LO-phonon stage. In this way they reduced the thermal backfilling of the lower state of the laser transition and obtained laser action up to $116 \mathrm{~K}$ in pulsed operation and $53 \mathrm{~K}$ in continuous wave at $3.6 \mathrm{THz}(\lambda=82 \mu \mathrm{m})$.

In this work we study the effect of a strong magnetic field applied parallel to the growth axis on the $\mathrm{THz}$ laser presented in ref. ${ }^{8}$. Under an applied magnetic field, each subband $|i\rangle$ splits into a ladder of Landau levels (LLs) $|i, n\rangle$ with energies:

$$
E_{i, n}=E_{i}+(n+1 / 2) \hbar \omega_{c}
$$

where $n$ is an integer and $\omega_{c}=e B / m^{\star}$ is the cyclotron frequency ( $e$ is the electron charge and $m^{\star}$ the energy dependent electron effective mass). The emitted power oscillates as a function of the magnetic field. These oscillations have already been observed in several mid- and far-infrared QCLs ${ }^{9,10}$ and ascribed to the fact that the lifetime of the upper state of the laser transition oscillates with $B$. We will show that our $\mathrm{THz}$ QCL presents a further series of oscillations, corresponding to a LO-phonon emission from hot electrons thermally distributed in the LLs of the upper subband of the laser transition. We will show that this effect is consistent with an electronic temperature $T_{e}=T_{l}+150 \mathrm{~K}$, where $T_{e}$ is the electronic temperature and $T_{l}$ the lattice one.

The QCL active region consists of 120 periods of a GaAs/ $/ \mathrm{Al}_{0.15} \mathrm{Ga}_{0.85} \mathrm{As}$ sequence grown on a GaAs semiinsulating substrate as described in reference ${ }^{8}$. In Fig. 1 we show the band diagram of one period of the active region under an electric field of $4 \mathrm{kV} / \mathrm{cm}$, obtained using a selfconsistent Schrodinger-Poisson calculation. The square moduli of the relevant wave functions are also shown, using the same indices for the various levels as in reference ${ }^{8}$. The ground level of the two large coupled quantum wells forming the LO-phonon extraction stage is labeled $|1\rangle$ and its first excited level is aligned resonantly with the extraction miniband, giving rise to levels labeled $|3\rangle$ to $|7\rangle$. Finally, state $|9\rangle$ is the isolated level which is created inside the minigap in the thin well adjacent to the injection barrier and is resonant with state $\left|1^{\prime}\right\rangle$ of the previous period. The radiative transition occurs between levels $|9\rangle$ and $|7\rangle\left(E_{97}=15 \mathrm{meV}\right)$. The energy separation $E_{71}$ is calculated to be $32 \mathrm{meV}$, close to ( $36 \mathrm{meV}$ ). This large energy separation was designed to reduce the lower state lifetime by allowing electron-LO phonon scattering.

Lasers were processed into $160 \mu \mathrm{m}$ wide and $1.5 \mathrm{~mm}$ long ridge waveguides. They were mounted inside an insert at the center of a superconducting coil where a magnetic field $B$ up to $15 \mathrm{~T}$ could be applied parallel to the growth axis. Light emitted by the laser was focused onto a composite silicon bolometer placed $5 \mathrm{~cm}$ below the device. The variation of the output power $P$ as a function of $B$ at $T=2 \mathrm{~K}$ is shown in Fig. 2(a). The laser was driven in pulsed mode with a $0.005 \%$ duty cycle and $200 \mathrm{~ns}$ pulse-width with an envelope of $90 \mathrm{~Hz}$ 


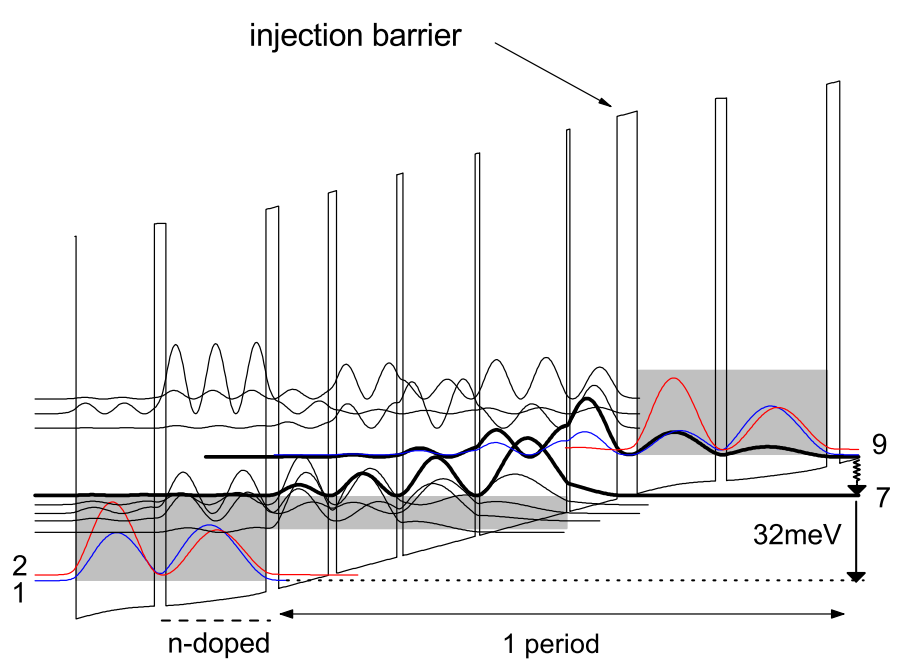

FIG. 1: Self-consistent solution of coupled Schrodinger an Poisson's equations for one period of the structure. Layer sequence starting from injection barrier is as follows: 42/100/7/183/10/152/13/127/17/105/27/211/24/165 (underlined layer is $\mathrm{Si}$ doped at $3.8 \times 10^{16} \mathrm{~cm}^{-3}$, figures in bold type are $\mathrm{Al}_{0.15} \mathrm{Ga}_{0.85} \mathrm{As}$ layers).

and the dependence $P(B)$ was obtained by using a standard lock-in technique.

A first series of pronounced minima is observed for magnetic field values such that $E_{97}=n \hbar \omega_{c}$, where $n=1,2,3 \ldots$ (solid arrows) in fig. 2(a). Indeed, when this condition is fulfilled, an additional relaxation path opens for electrons in the LL $|9,0\rangle$ to elastically scatter into the LLs $|7, n\rangle$. This leads to a strong decrease of the population inversion between $|9,0\rangle$ and $|7,0\rangle$ and hence of the output power $P$. Fig. 2(b) shows the Landau levels $|9, n\rangle$ and $|7, n\rangle$ as functions of the magnetic field. Dashed vertical lines are used to indicate the values of the magnetic field, for which elastic scattering is possible between $|9,0\rangle$ and $|7, n\rangle$. From fig. 1 we can see that the relevant wavefunctions are delocalized over several quantum wells. As a consequence, the wavefunctions of the subbands $|9\rangle$ and $|7\rangle$ are not negligible for at least 4 interfaces. We believe that in this situation the relevant elastic scattering process is essentially the interface roughness ${ }^{11,12}$. Following ref. ${ }^{11}$, we calculated the lifetime of the upper state of the laser transition $\tau_{9,0}$ as a function of the magnetic field (fig. 2(c)).

In order to reproduce all the experimental features, the lifetime has been obtained by considering the relaxation via the interface roughness scattering from $|9,0\rangle$ Landau level to the Landau levels of all the subbands of the extraction miniband:

$$
\frac{1}{\tau_{9,0}^{\text {roughness }}}=\sum_{n=0}^{80}\left(\frac{1}{\tau_{9,0-7, n}^{\text {roughness }}}+\sum_{i=3}^{6} \frac{1}{\tau_{9,0-i, n}^{\text {roughness }}}\right)
$$

For the calculation, we used a Gaussian autocorrelation of the roughness, with an average height $\Delta=1.5 \AA$ and a correlation length $\Lambda=60 \AA$, as in ref. ${ }^{11}$. The calculated upper state lifetime as a function of magnetic field is shown in figure 2(c) and we observe that the minima indicated by the solid arrows in fig. 2(a) can be very well reproduced. In particular, the laser

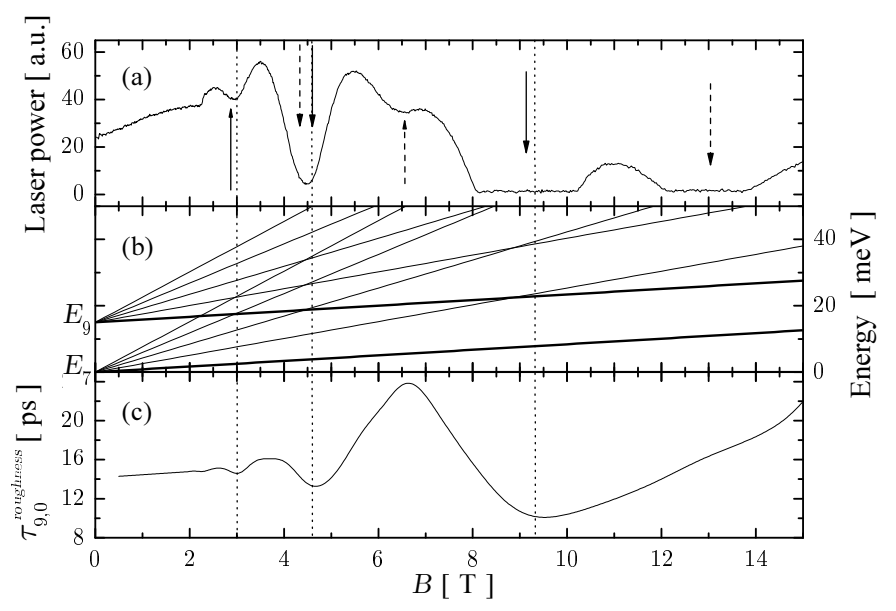

FIG. 2: (a) Emitted power as a function of the magnetic field under a constant current $J=236 \mathrm{~A} . \mathrm{cm}^{-2}$. (b) Plot of the laser Landau levels. note the crossing at $9 \mathrm{~T}, 4.5 \mathrm{~T}, 3 \mathrm{~T}$. (c) Interface roughness lifetime as a function of the magnetic field, calculating by taking into account transitions from $|9,0\rangle$ to $|7, n\rangle,|6, n\rangle,|5, n\rangle,|4, n\rangle,|3, n\rangle$.

switches off between 8 and $10 \mathrm{~T}$ due to the fundamental elastic resonance $\left(E_{90}=E_{71}\right)$. We considered a Gaussian broadening of the Landau levels, with a width $\delta=2 \mathrm{meV}$. This value was obtained by comparing figs. 2(a) and 2(c) and assuming that the features indicated by the arrows are mainly due to the magnetic field dependence of the upper state lifetime.

From the comparison betweens figs. 2(a) and 2(c), we can see that the minimum at $6.5 \mathrm{~T}$ cannot be ascribed to the elastic scattering process described before. Furthermore, we can see that the laser switches off between $12 \mathrm{~T}$ and $14 \mathrm{~T}$, after the fundamental elastic resonance. Resonances with the excited Landau levels of the subbands $|i\rangle$, with $i<7$, cannot be responsible for these features: the overlap between the wavefunctions of subbands $|9\rangle$ and $|i\rangle$ at the interfaces is lower than between $|9\rangle$ and $|7\rangle$. As a consequence, the lifetimes related to these processes are longer. Moreover, the features at $6.5 \mathrm{~T}$ and $13 \mathrm{~T}$ cannot be explained by resonant electron-electron scattering effect (Auger process), which has recently been observed in a QCL based on an intersubband transition between states of a large quantum well ${ }^{13}$. Indeed, in the sample investigated here, such a process would occur when $2 \times E_{97}=30 \mathrm{meV}=\hbar \omega_{c}$ corresponding to $B=18 \mathrm{~T}$, beyond our investigated field range. The series of minima of the emitted power that cannot be explained by elastic resonances between the $|90\rangle$ Landau level and the excited Landau levels of the lower subbands is indicated in fig. 2(a) by dashed arrows.

Fig. 3(a) shows the excited Landau levels of subband 9, as well as the fundamental Landau level of subband 7. The dashed line indicates the first LO-phonon replica of $|7,0\rangle$ Landau level. We notice that at $13 \mathrm{~T},|9,1\rangle$ the first excited Landau level of the upper subband has a phonon resonance with $|7,0\rangle$ the fundamental Landau level of the lower subband: $E_{91}-E_{70}=\hbar \omega_{L O}$. We will show in the following that this relaxation mechanism is a result of hot electrons in the injector that decrease the gain in the structure. 


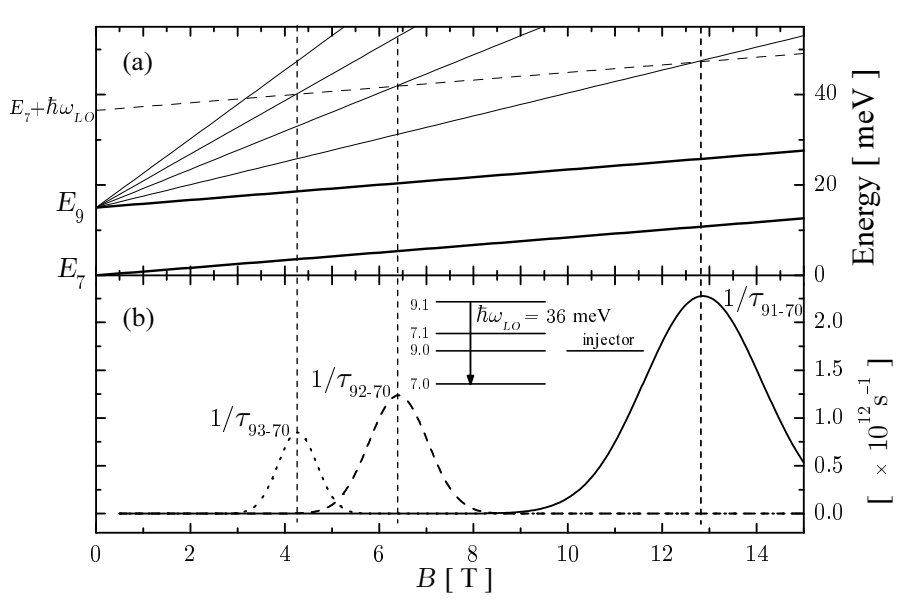

FIG. 3: (a) Fan chart of laser Landau levels (solid lines) and level $|7, n\rangle+\hbar \omega_{L O}$ (dashed line). Note the crossing at $13 \mathrm{~T}, 6.5 \mathrm{~T}, 4.3 \mathrm{~T}$. LO-phonon scattering rate as a function of the magnetic field, calculated by taking into account transitions $|9,1\rangle$ to $|7,0\rangle$ in solid line, $|9,2\rangle$ to $|7,0\rangle$ in dashed line and $|9,3\rangle$ to $|7,0\rangle$ in dotted line. (inset) Four levels system at $13 \mathrm{~T}$.

After the fundamental elastic resonance at $9 \mathrm{~T}$, the system is in the extreme quantum limit. In order to understand why the laser switches off at $13 \mathrm{~T}$, we compare the gain in the structure at $13 \mathrm{~T}$ (when the laser is off) and at $14 \mathrm{~T}$ (when the laser is on) by assuming that it is possible to apply the same system of rate equations. We consider a four level system, as shown int the inset fig. 3(b), coupled with an injector state. We assume that the electronic temperature $T_{e}$ in the injector is greater than the lattice temperature $T_{l}\left(T_{e}=T_{l}+150 \mathrm{~K}\right)$; this assumption is consistent with the results of photoluminescence experiments on $\mathrm{THz} \mathrm{QCLs}^{14}$ and with those obtained to reproduce the temperature dependence of the threshold current density in the same structure ${ }^{8}$. We assume a Boltzmann distribution of the electrons in the injector, and we calculate the population inversion below threshold using a complete set of rate equations for levels $|9\rangle$ and $|7\rangle$ :

$$
\begin{aligned}
& {\left[N_{9}-N_{7}\right]_{B>9 T} \approx \frac{J}{e}\left[\tau_{90}\left(1-\frac{\tau_{e s c}}{\tau_{90-70}}\right)\right.} \\
& \left.+e^{-\left(E_{91}(B)-E_{90}(B)\right) /\left(k_{B} T_{e}\right)} \times \tau_{91}\left(1-\frac{\tau_{e s c}}{\tau_{91-70}(B)}\right)\right]
\end{aligned}
$$

where $\tau_{\text {esc }}$ is the escape time from the lower state of the radiative transition, $k_{B}$ is the Boltzmann constant and $J$ the density current in the device. The scattering rate of level $|9,1\rangle$, $1 / \tau_{91 \rightarrow 70}$, is calculated by only taking into account the LO- phonon emission and is shown by a solid curve in fig. 3(b). We assume that $\tau_{\text {esc }}$ and $\tau_{90 \rightarrow 70}$ are constant with magnetic field as a consequence of the extreme quantum limit.

Using cavity losses $\alpha=16.2 \mathrm{~cm}^{-1}$, the application of eq. 3 at $14 \mathrm{~T}$ at threshold allows us to extract the value of the population inversion necessary to achieve a laser action. From the comparison between this value and $\left[N_{9}-N_{7}\right]$ at $B=13 \mathrm{~T}$, $J=236 \mathrm{~A} / \mathrm{cm}^{2}$, we obtain a limiting condition on $\tau_{e s c}$ for the inhibition of the laser emission. We obtain that if $\tau_{e s c}>$ $20 \mathrm{ps}$, the condition for laser action is not satisfied. If we calculate $\tau_{e s c}$ by assuming the relaxation via the phonon stage as the main relaxation mechanism involved, we find an escape time of 22 ps. As a consequence, the relaxation of hot electrons by the emission of an LO phonon may be invoked as the mechanism responsible for the second series of oscillations in the $P(B)$ spectrum. Fig. 3(b) also shows in dashed lines the scattering rates for LO-phonon emission, corresponding to $|9,2\rangle \rightarrow|7,0\rangle$ transition and $|9,3\rangle \rightarrow|7,0\rangle$ transition: they are peaked at the minima of the $P(B)$ curve shown in figure 2(a) (dashed arrows).

The two sets of power oscillations observed can now be summarized by the following equations, showing that a reduction in the population inversion is observed when:

$$
E_{90}=E_{70}+m \hbar \omega_{c} ; \text { with } \mathrm{m}=1,2,3 \ldots
$$

and

$$
E_{90}+n \hbar \omega_{c}=E_{70}+\hbar \omega_{L O} ; \text { with } \mathrm{n}=1,2,3 \ldots
$$

reproducing all the minima observed in the $P(B)$ characteristics.

In conclusion, we have investigated the output power dependence under applied magnetic field on a QCL design with a LO-phonon extraction stage. Two distinct series of oscillations are identified corresponding to Landau level resonances between the upper and lower laser transition states. The first series correspond to an elastic scattering from the fundamental Landau level of the upper subband. The second, which has not been previously observed, is associated to the relaxation of hot electrons via the emission of an LO-phonon.

The Laboratoire Pierre Aigrain is a "Unite Mixte de Recherche" (UMR 8551) between Ecole Normale Supérieure, the CNRS, the University Pierre et Marie Curie (Paris 6) and the University Denis Diderot (Paris 7). We would like to thank R. Ferreira for very valuable and fruitful discussions.
* Electronic address: louis-anne.devaulchier@lpa. ens.fr

${ }^{1}$ M. Rochat, L. Ajili, H. Willenberg, J. Faist, H. Beere, G. Davies, E. Linfield, and D. Ritchie, Appl. Phys. Lett. 81, 1381 (2002).

${ }^{2}$ R. Kohler, A. Tredicucci, F. Beltrann, H. Beere, E. Linfield, A.
Davies, D. Ritchie, R. Iotti, and F. Rossi, Nature 417, 156 (2002).

${ }^{3}$ G. Scalari, L. Ajili, J. Faist, H. Beere, E. Linfield, D. Ritchie, and G. Davies, Appl. Phys. Lett. 82, 3165 (2003).

${ }^{4}$ S. Barbieri, J. Alton, H. Beere, J. Fowler, E. Linfield, and D. Ritchie, Appl. Phys. Lett. 85, 1674 (2004). 
${ }^{5}$ B. Williams, S. Kumar, H. Callebaut, Q. Hu, and J. Reno, Appl. Phys. Lett. 82, 5142 (2003).

${ }^{6}$ R. Kohler, A. Tredicucci, C. Mauro, F. Beltram, H. Beere, E. Linfield, A Davies, and D. Ritchie, Appl. Phys. Lett. 84, 1266 (2004).

7 S. Kumar, B. Williams, S. Kohen, Q. Hu, and J. Reno, Appl. Phys. Lett. 84, 2494 (2004).

${ }^{8}$ G. Scalari, N. Hoyler, M. Giovannini, and J. Faist, Appl. Phys. Lett. 2, 86 (2005).

9 J. Alton, S. Barbieri, J. Fowler, H. Beere, J. Miscat, E. Linfield, D. Ritchie, G. Davies, R. Khler, and A. Tredicucci, Phys. Rev. B 68, 081203 (2003).

${ }^{10}$ G. Scalari, S. Blaser, L. Ajili, J. Faist, H. Beere, E. Linfield, D.
Ritchie, and G. Davies, Appl. Phys. Lett. 83, 3453 (2003).

11 A. Leuliet, A. Vasanelli, A. Wade, G. Fedorov, D. Smirnov, G. Bastard, and C. Sirtori, Phys. Rev. B 73, 085311 (2006).

12 G. Scalari, C. Walther, L. Sirigu, M.L. Sadowski, N. Hoyler, M. Giovannini, H. Beere, D. Ritchie, and J. Faist, unpublished (2007).

13 G. Scalari, S. Blaser, J. Faist, H. Beere, E. Linfield, D. Ritchie, and G. Davies, Phys. Rev. Lett. 93, 237403 (2004).

${ }^{14}$ M.S. Vitiello, G. Scamarcio, V. Spagnolo, B. Williams, S. Kumar, Q. Hu, and J. Reno, Appl. Phys. Lett. 86, 111115 (2005). 